\title{
Rapid prototyping of magnetic tunnel junctions with focused ion beam processes
}

\author{
A Persson, $G$ Thornell and $H$ Nguyen \\ Ångström Space Technology Centre, Dept. of Engineering Sciences, Uppsala University, \\ Sweden \\ E-mail: anders.persson@angstrom.uu.se
}

This is an author-created, un-copyedited version of an article accepted for publication in Journal of micromechanics and microengineering. IOP Publishing Ltd is not responsible for any errors or omissions in this version of the manuscript or any version derived from it. The definitive publisher authenticated version is available online at doi: 10.1088/09601317/20/5/055039.

\begin{abstract}
Submicron sized Magnetic tunnel junctions (MTJs) are most often fabricated by time-consuming and expensive e-beam lithography. From a research and development perspective, a short lead time is one of the major concerns. Here, a rapid process scheme for fabrication of micrometer size MTJs with focused ion beam processes is presented. The magnetic properties of the fabricated junctions is investigated in terms of magnetic domain structure, tunnelling magnetoresistance (TMR) and coercivity, with extra attention to the effect of Ga implantation from the ion beam. In particular, the effect of the implantation on the minimum junction size and the magnetization of the sensing layer are studied. In the latter case, magnetic force microscopy and micromagnetic simulations, with the Object Oriented Micromagnetic Framework (OOMMF), are used to study the magnetization reversal. The fabricated junctions show considerable coercivity both along their hard and easy axes. Interestingly, the sensing layer exhibit two remanent states: one with a single and one with a double domain. The hard axis TMR loop has kinks at about $\pm 20 \mathrm{mT}$ which is attributed to a non-uniform lateral coercivity, where the rim of the junctions, which is subjected to Ga implantation from the flank of the ion beam, is more coercive than the unirradiated centre. The width of the coercive rim is estimated to $160 \mathrm{~nm}$ from the hard axis TMR loop. The easy axis TMR loop shows more coercivity than an unirradiated junction and, this too, is found to stem from the coercive rim, as seen from the simulations. It is concluded that the process scheme has three major advantages. Firstly, it has a high lateral and depth resolution - the depth resolution is enhanced by end point detection - and is capable of making junctions of sizes down towards the limit set by the width of the irradiated rim. Secondly, the most delicate process steps are preformed in unbroken vacuum enabling the use of materials prone to forming oxides in the MTJ film stack. Thirdly, the scheme is both uncomplicated and quick and makes it possible to go from design to characterization in the order of hours.
\end{abstract}

\section{Introduction}

Magnetic tunnel junctions (MTJs) are used in, e.g., read heads for computer hard drives [1], Magnetic Random Access Memories (MRAMs) [2], and highly sensitive magnetic sensors [3]. These junctions are characterized by high sensitivity and are manufactured using standard micro- and nano-structuring technologies. The films in an MTJ stack are typically from a few hundred down to a less than a nanometre thick, wherefore an etch stop accuracy in the nanometre range is desirable in the mentioned applications. A lateral junction dimension of less than $1 \mu \mathrm{m}$ is preferred for high-density MRAMs and for good read head performance [4-6]. Patterning of large series of MTJs in this size range is typically made using a combination of UV and e-beam lithography [7] followed by ion milling [8]. For research and development, where pattern designs and dimensions often require several adjustments, and evaluation is iterative, these methods are less suitable. Instead, lead time reduction is of major concern, and rapid prototyping is a powerful means of progress. 
As an alternative to, for instance, e-beam lithography, focused ion beam (FIB) technology can be used in this respect. In a high-end FIB equipment, both direct ion milling [9] and ion- or electron-assisted chemical vapour deposition (IACVD and EACVD, respectively) [10] can be performed. Usually, the technique also has adequate accuracy and resolution for making MTJs in the sub micrometer range [11].

However, FIB patterning suffers from some disadvantages - the most important one being the potentially harmful ion implantation [12-14]. The effects of beam-associated Ga implantation in the $\mathrm{CoFeB}$ and $\mathrm{CoFe}$ soft magnetic alloy layers, used also here, have previously been studied and presented [15].

In this paper, FIB-based rapid prototyping of MTJs is presented. Contrary to most other efforts in the field, the effect of the individual processing steps are studied both in situ and ex situ. In particular, the resulting MTJs' tunnelling magnetoresistance (TMR) and coercivity are related to the Ga implantation. Also, the minimum junction size is approximated under the assumption that the $\mathrm{Ga}$ implantation will be the limiting factor. Since the size of the implantation damaged part of the junctions is too small to study with conventional methods, it is estimated from the TMR measurements.

\section{Methods}

The MTJ stack used in this study was, top-down: $7 \mathrm{Ru} / 10 \mathrm{Ta} / 3 \mathrm{Co}_{60} \mathrm{Fe}_{20} \mathrm{~B}_{20} / 0.3 \mathrm{Mg} / 1.1 \mathrm{MgO} / 3$ $\mathrm{Co}_{60} \mathrm{Fe}_{20} \mathrm{~B}_{20} / 0.8 \mathrm{Ru} / 2.5 \mathrm{Co}_{70} \mathrm{Fe}_{30} / 20 \mathrm{PtMn} / 5 \mathrm{Ta} / 30 \mathrm{CuN} / 5 \mathrm{Ta}$, where the capital numbers are thicknesses in $\mathrm{nm}$, subscripts are contents in at. \% and the PtMn is nominally equiatomic. It was deposited on a thermally oxidized Si (100) wafer by Singulus Technologies AG, Germany, using a ten-target TIMARIS sputter. The $\mathrm{MgO}$ barrier was made by oxidising the sputtered $\mathrm{Mg}$ layer in 133 Pa oxygen atmosphere for $1200 \mathrm{~s}$. The stack was annealed at $360^{\circ} \mathrm{C}$ for $2 \mathrm{~h}$ in an applied field of $1 \mathrm{~T}$, and had a TMR of $163 \%$ and a resistance area product of $8.4 \Omega \mu \mathrm{m}^{2}$. Here, the top CoFeB layer was the sensing layer, and the synthetic antiferromagnetic (SAF) $\mathrm{CoFeB} / \mathrm{Ru} / \mathrm{CoFe}$ layers were pinned by ferromagnetic-antiferromagnetic exchange coupling to the PtMn layer. The sensing layer had a fieldinduced uniaxial anisotropy with the easy axis parallel to the pinning direction of the SAF layers.

Some of the process equipment used here could not handle samples larger than a few centimetres, wherefore the wafers were diced into $10 \times 10 \mathrm{~mm}$ chips using a vertical blade dicing saw (Disco DAD 361, Disco Corp, Japan). During dicing, the stack was protected by a $10 \mu \mathrm{m}$ thick resist layer (AZ4562, AZ Electronic Materials, Luxembourg). In order to minimize the milling with FIB, the chips were pre-patterned using Ar ion milling (Sputnik, Albanova Nanofabrication Facility, Sweden), creating 36 rectangular $(200 \times 50 \mu \mathrm{m})$ sample sites, here simply referred to as samples. The etch mask was a $150 \mathrm{~nm}$ thick aluminium film deposited by magnetron sputtering (CS 730S, von Ardenne, Germany) in an $800 \mathrm{~W}$ Ar plasma under a process pressure of $0.44 \mathrm{~Pa}$, and patterned using standard UV lithography.

The resolution and milling rate of FIB depend on the ion beam current, with a high current resulting in low resolution but high milling rate, and vice versa. Therefore, the beam current was adjusted for rough and fine milling to reduce the total milling time. The milling depth was estimated in situ by End Point Detection (EPD) monitoring the current through the sample stage, in the FIB (Strata DB235, FEI Company, USA). As this current varies with the material immediately targeted by the beam, EPD can, given a known stack composition, be used to assess the milling depth. Since the milling rate depends both on the beam current, which may vary from time to time depending, for example, on the quality of the Ga source, and the very material being milled, the EPD is more reliable than using deduced reckoning based on the milling time.

The patterns used in the FIB were created using the Patterns 1.04 software which converts bitmap images into $1024 \times 1024$ pixel large milling files for the FIB software (FEI xp, FEI, USA). Before converting the bitmaps to milling files, the beam overlap and dwell time were set. Ion beam settings and process parameters for rough and fine milling, as well as for deposition, are presented in table 1 . The acceleration voltage was $30 \mathrm{kV}$ throughout the experiment. 
Table 1. Ion beam settings and process parameters. The acceleration voltage was $30 \mathrm{kV}$ in all modes.

\begin{tabular}{lccccc}
\hline Mode & $\begin{array}{c}\text { Beam current } \\
{[\mathrm{pA}]}\end{array}$ & $\begin{array}{c}\text { Beam overlap } \\
{[\%]}\end{array}$ & $\begin{array}{c}\text { Dwell time } \\
{[\mu \mathrm{s}]}\end{array}$ & $\begin{array}{c}\text { Dose } \\
{\left[\mathrm{Ga}^{+} \mathrm{cm}^{-2}\right]}\end{array}$ & $\begin{array}{c}\text { Dose rate } \\
{\left[\mathrm{Ga}^{+} \mathrm{cm}^{-2} \mathrm{~s}^{-1}\right]}\end{array}$ \\
\hline Rough milling* & 3000 & 50 & 1 & $1.1 \times 10^{17}$ & $2.0 \times 10^{14}$ \\
Fine milling & 100 & 50 & 1 & $1.1 \times 10^{17}$ & $4.4 \times 10^{13}$ \\
Deposition & 100 & 0 & 0.2 & $2.9 \times 10^{16}$ & $4.4 \times 10^{13}$ \\
\hline
\end{tabular}

$*$ A dose of $1.7 \times 10^{17} \mathrm{Ga}^{+} \mathrm{cm}^{-2}$ and a dose rate of $1.6 \times 10^{15} \mathrm{Ga}^{+} \mathrm{cm}^{-2} \mathrm{~s}^{-1}$ was used for milling of the alignment marks.

In this study, six pre-patterned samples, labelled S1-S6, were used. After each FIB process step, one sample was left unchanged for subsequent investigation. Thus, samples S1 to S6 were finalized at process steps corresponding, respectively, to (b) to (g) in the process scheme, figure 1, and only in sample S6, all steps were accumulated, resulting in a full MTJ structure - a set of 48 MTJs to be precise. Each step of the process was monitored by EPD and SEM.

The patterning process began with defining an alignment mark on S1-S6 (figure $1 \mathrm{~b}$ ) for the rough and fine milling steps. This was done with the rough milling settings and, assisted by the EPD, the milling was terminated in the $\mathrm{SiO}_{2}$ part of the substrate. The alignment marks limited the area that had to be fine-patterned and capped in order to isolate the top contact, and thereby reduced the total process time significantly.

Contouring of the MTJ was made successively on S2-S6 with the fine milling settings (figure $1 \mathrm{c}$ ). To limit the radiation dose in the active parts of the film stack, the MTJ patterns were aligned using the selected area scan function in the FIB software. The milling was stopped in the PtMn layer using EPD. The junction patterns of S3-S6 were capped with $\mathrm{SiO}_{2}$ (figure $1 \mathrm{~d}$ ) using IACVD with an ethyl silicate $\left(\mathrm{C}_{8} \mathrm{H}_{20} \mathrm{O}_{4} \mathrm{Si}\right)$ precursor emitted from a needle gas injector positioned approximately $100 \mu \mathrm{m}$ above the beam-sample intersection. Water vapour from a magnesium hydrate sulphate salt was added to improve the deposit's quality and increase the deposition rate. The chamber pressure was $3.5 \times 10^{-3} \mathrm{~Pa}$ during deposition. The advantage of this step was that the pattern was the same as for contouring the junctions and therefore self-aligning since the masked area was milled in the first case and coated in the second. The contour of an MTJ endured an irradiation dose of $3 \times 10^{16} \mathrm{Ga}^{+} \mathrm{cm}^{-2}$ in this coating step.

Next, the rest of the pre-patterned areas of S4-S6 were milled down to the second Ta layer (figure $1 \mathrm{e}$ ) using the rough milling settings. Again, alignment of the patterns was done using the selected area scan, and the milling depth was monitored using EPD. Finally, the sidewalls of the bottom contacts of $\mathrm{S} 5$ and $\mathrm{S} 6$ were capped (figure $1 \mathrm{f}$ ) using IACVD of $\mathrm{SiO}_{2}$ as before.

Although platinum leads could be deposited in the FIB system using a trimethylmethylcyclopentadienyl-platinum $\left(\mathrm{C}_{9} \mathrm{H}_{16} \mathrm{Pt}\right)$ precursor [16] in a manner similar to that for the $\mathrm{SiO}_{2}$ capping, the leads and pads for sample S6 were patterned using standard UV lithography and lift-off of an Al film equal to that of the etch mask. To improve adhesion, the chip was pre-treated in a $50 \mathrm{~W}$ $\mathrm{O}_{2}$ plasma (TePla 300, TePla Inc., USA) for 120 s. For the subsequent magnetic analysis, S6 was connected to a printed circuit board, using wire bonding (4526, Kulicke \& Soffa, USA). 
(a)

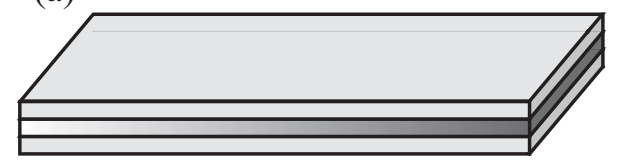

(b)



(c)

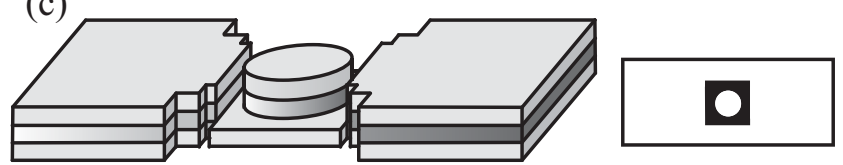

(d)


Figure 1. Scheme of the FIB process steps: pre-patterning (a), alignment mark milling (b), MTJ contour milling (c), capping (d), bottom contact milling (e), bottom contact capping (f), and contact leads deposition (g). To the right are the bitmaps used for creating the corresponding patterns. (In this experiment, a more detailed pattern with 48 MTJs was used)

The progress and quality of the process was monitored using the in-situ scanning electron microscopy (SEM) and EPD functions of the FIB. Prior to the S1-S6 manufacturing, the relationship between EPD current and depth was acquired by milling $400 \mu \mathrm{m}^{2}$ squares with the fine milling settings (table 1) to different depths, and identifying the PtMn layer and $\mathrm{SiO}_{2}$ substrate using an atomic force microscope (AFM) (XE150, PSIA, USA). The AFM was also used to investigate samples S1-S5. In addition, sample S2 was studied with magnetic force microscopy (MFM) (Dimension 3100, Veeco Instruments, USA) to investigate the effects of Ga implantation on the domain structure of the junction sensing layers. Finally, the TMR loop of S6 (the full MTJ) was investigated using a four-point current probe (Model 2400, Keithley Instruments, USA) and an electromagnet connected to a computer-controlled current source (BOP 10-75MG, KEPCO Inc., USA). This set up was used to measure the resistance of the sensor as a function of applied magnetic field at room temperature, with a constant bias current of $5 \mathrm{~mA}$ and the magnetic field applied along both the hard and the easy axis of the MTJ. Sensors from the same MTJ stack, fabricated with a more time-consuming process using Ar instead of Ga milling [15], were also studied in a similar way to be used as a reference. 


\section{Simulations}

The TMR of an MTJ is governed by, e.g., the spin polarization of the ferromagnetic electrodes, the quality of the barrier, the bias voltage, the resistance and area of the junction. However, for a single domain MTJ from a given TMR layer structure and for use of this as a magnetometer, the change in resistance under a field applied along the hard axis is empirically given by:

$$
\Delta R=(T R A / 2 W h) \cos \left(\theta_{s}-\theta_{p}\right),
$$

where $T$ is the TMR, $R$ the resistance, $A$ the area of the junction, $\theta_{s^{-}} \theta_{p}$ the angle between the magnetization of the sensing and pinning layers, and $W h$ (width times height) a geometry factor defining the shape anisotropy of the sensing layer [3].

Here, the magnetization reversal of the sensing layer was studied by micromagnetic simulations using the Object oriented micromagnetic framework (OOMMF) [17]. The sensing layer was modelled as an ellipse with a thickness of $5 \mathrm{~nm}$, and minor and major axes of $1 \mu \mathrm{m}$ and $2 \mu \mathrm{m}$, respectively, according to the lateral dimensions of the manufactured MTJs. Single layer $5 \times 5 \times 5 \mathrm{~nm}$ cells were used for meshing the simulation volume, and, for simplicity, both crystalline anisotropy and coupling effects from the SAF layers were disregarded. (With an actual thickness of $3 \mathrm{~nm}$, cells of $3 \times 3 \times 3 \mathrm{~nm}$ would better represent the manufactured MTJs, but for verification of the design, it is sufficient to use a coarser mesh.) The CoFeB was modelled with a saturation magnetization, $M_{s}$, of $930 \mathrm{kA} / \mathrm{m}$, an exchange coefficient, $A_{e}$, of $12 \mathrm{pJ} / \mathrm{m}$, and a damping coefficient, $\alpha$, of 0.15 [18]. The magnetization of the sensing layer was simulated under the influence of a magnetic field directed along the major axis, which was swept between positive and negative saturation. The magnetization of the cells was acquired at each simulation stage and post-processed, using Eq. 1, to record the simulated TMR loop. The pinning layer was assumed to have a constant and uniform magnetization directed along the sensing layer major axis.

\section{Results \& Discussion}

From the recorded curve of EPD current vs. milling dose, figure 2, and supplementary AFM measurements, the location of the PtMn layer and the $\mathrm{SiO}_{2}$ substrate are readily obtained. It was confirmed that, doses of $5.1 \times 10^{16} \mathrm{Ga}^{+} \mathrm{cm}^{-2}$ and $7.6 \times 10^{16} \mathrm{Ga}^{+} \mathrm{cm}^{-2}$ corresponded to depths of $31 \mathrm{~nm}$ and $95 \mathrm{~nm}$, respectively.

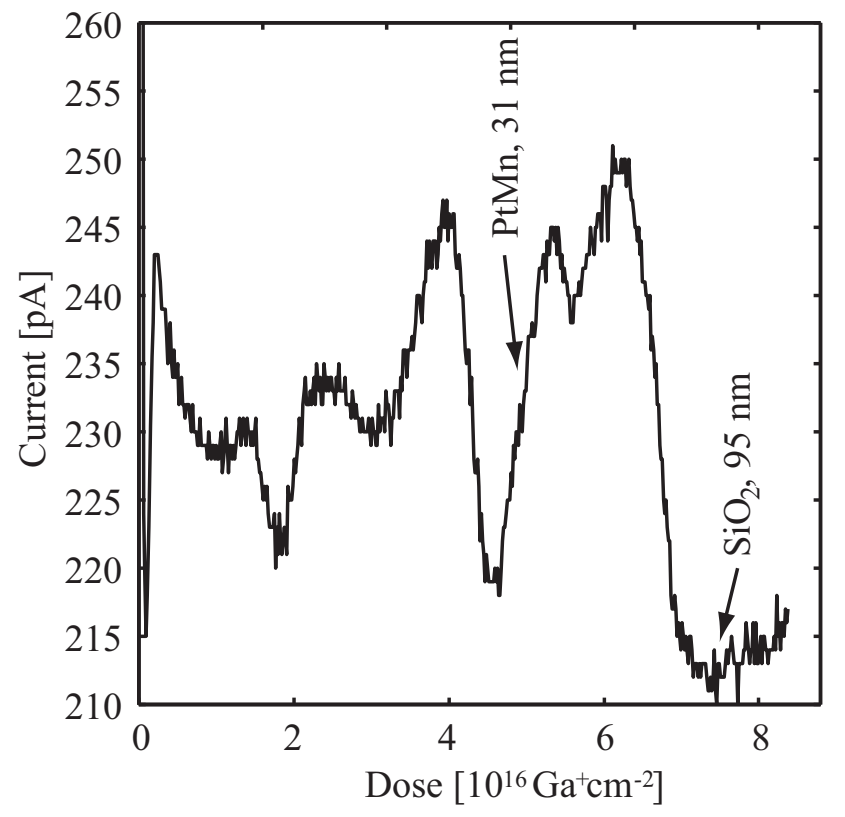

Figure 2. Modified EPD curve for the MTJ stack showing the PtMn and $\mathrm{SiO}_{2}$ layers.

The fabrication process showed good resolution and accuracy for making MTJs in the desired size range. The pre-patterning of the samples, made outside the FIB, allowed for contacting of multiple samples using UV lithography and lift-off. However, the entire process could be performed using FIB 
alone, but if so, preferably on smaller samples due to the relatively low milling rate of the ion beam. Each step of the manufacturing was monitored by EPD and SEM.

The vertical lines in the AFM image of contoured MTJs, figure 3 (left), are caused by uneven spacing of the milling points in the pattern converted in the Patterns software. The MFM image of the same MTJs, figure 3 (right), was recorded after the sensing layer had relaxed from saturation with a magnetic field directed $90^{\circ}$ to the antiferromagnetic pinning direction of the CoFeB SAF layer.
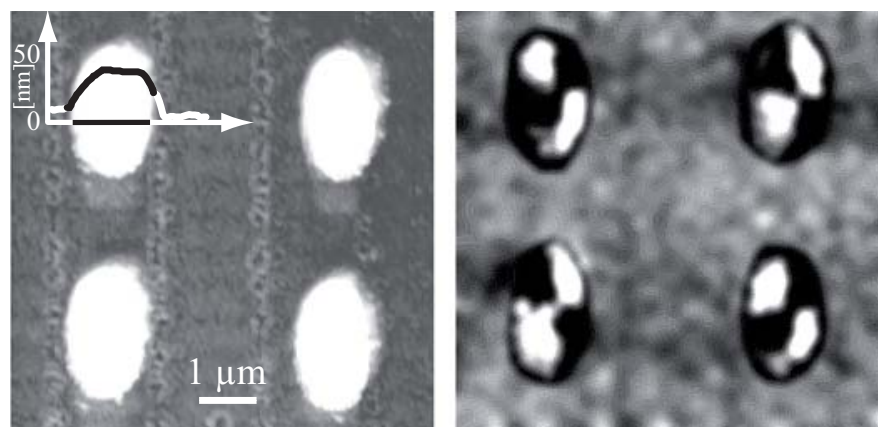

Figure 3. AFM (left) and MFM (right) image of the contoured MTJs. Superimposing one of the junctions (top left) is a height profile.

The main advantages with the FIB processing were the possibility of monitoring the milling depth in situ by EPD, figure 2, and using the same pattern mask for milling and capping the sensors, making alignment without direct Ga irradiating the MTJs possible. Moreover, the fact that the process was completely performed in vacuum enabled the use of materials prone to forming oxides in the MTJ stack.

However, the capping of the bottom contact of the MTJs, figure 1 (d), had the side effect that also the top of the MTJs was coated with a thin layer of $\mathrm{SiO}_{2}$ isolating the top electrode. This effect has also been observed in, e.g., [11], and is due to the Gaussian shape of the ion beam, the scattering of ions in the plasma and, to less extent, the unassisted $\mathrm{CVD}$ of $\mathrm{SiO}_{2}$ outside the irradiated zone. To solve this problem, an additional step of gentle Ar ion milling to remove the $\mathrm{SiO}_{2}$ from the top of the MTJs, had to be added here before depositing the contacts. This extra step could be conveniently made along with the ex-situ UV lithography and lift-off, since keeping the sample under unbroken vacuum inside the FIB was no longer necessary. For an all-FIB prototyping solution, one could have deposited a Pt implantation barrier on top of the MTJs, using EACVD, before capping them, and then removed the excess $\mathrm{SiO}_{2}$ by $\mathrm{Ga}$ ion milling.

The MTJs fabricated showed considerable coercivity along both the magnetic easy and hard axis. Even though they had not been subjected to direct Ga irradiation, the Gaussian shape of the ion beam caused some implantation along the rim of the junctions during both milling and capping. The effects of a direct Ga irradiation to the MTJ stack used here, has shown that a dose of $10^{14} \mathrm{Ga}^{+} \mathrm{cm}^{-2}$ reduces the TMR of the junction by $60 \%$ and doubles the resistance area product, whereas the TMR was completely lost at a dose of $10^{16} \mathrm{Ga}^{+} \mathrm{cm}^{-2}$ [15]. Here, the dose required for milling and capping the MTJs was about $10^{17} \mathrm{Ga}^{+} \mathrm{cm}^{-2}$, and thus, the rim of the sensors was subjected to a damaging irradiation dose, giving the sensing layer a coercivity decreasing from the rim to the centre. As can be seen from the hard axis TMR loop, figure 4, this resulted in an unexpected magnetization reversal of the sensing layer. 




Figure 4. Hard axis TMR loop with illustrations of the rim vs. centre magnetization of the MTJ ellipses caused by the manufacturing process. The forward and reverse-going branches are indicated by arrows.

The magnetization of the sensing layers exhibits two remanent states: one with a single domain and one with a double domain, figure 3 and 4 . Apparently, the magnetization relaxes into the double domain state if the sensing layer is saturated and relaxed with a magnetic field applied $90^{\circ}$ to the pinning direction of the $\mathrm{CoFeB} \mathrm{SAF}$ layer. Correspondingly, the magnetization relaxes into the single domain state if the sensing layer is saturated in the opposite direction. This behaviour was not sensitive to misalignment of the hard axis and was assumed to stem from an imbalance in the SAF layers causing an asymmetric dipole coupling along, not only, the easy but also along the hard axis. This unbalance was caused by the shape of the ion beam, which was not exactly Gaussian, but it had an intensity distribution slightly elongated on one side. This can be seen from the height profile of the MTJ in figure 3, where the contour is slightly steeper on the right side than on the left. The asymmetric beam caused more implantation in one side of the MTJs, and the fringe field of the SAF layers therefore had a component in the hard axis direction. However, more experiments and simulations are required to fully understand this phenomenon.

The TMR loop had kinks at about $\pm 20 \mathrm{mT}$, figure 4, after which the magnetization of the sensing layer started to rotate more slowly. This was most likely due to the non-uniform coercivity of the sensing layer, where the magnetization of the softer centre rotated faster than that of the stiffer rim, due to the Ga implantation effect.

To estimate the area of the unirradiated centre, $A_{C}$, and the irradiated rim, $A_{R}$, the measured hard axis TMR loop, figure 4, is matched to equation 1, by assuming that the TMR and the resistance-area product are constant in both the centre and the rim. Furthermore, the measured TMR of the MTJs is assumed to be a superposition of the TMR loops of these two areas, figure 5. Here, the TMR loops of the centre and the rim are constructed by interpolation of the measured data, by assuming that the loop of the rim is dominant outside, and the loop of the centre is dominant inside the kinks. Now, the relationship between the areas, $A_{C}$ and $A_{R}$, can be found by integrating the right side of equation 1 , with $\theta_{s} \in[-\pi / 2, \pi / 2]$ and $\theta_{p}=0$, and the left side numerically using the interpolated loops, giving

$$
\int_{i}=T_{i} R_{i} A_{i} / W h
$$

where $\int$ is the numerical integral, the constants $T$ and $R$ are derived to $T_{R}=0.4 T_{C}$ and $R_{R}=8 R_{C}$ from [15] and the index $i$ is $C$ for centre and $R$ for rim. Here, the mean irradiation dose in the rim is supposed to be $10^{15} \mathrm{Ga}^{+} \mathrm{cm}^{-2}$ (assuming the dose to decline linearly from $10^{13} \mathrm{Ga}^{+} \mathrm{cm}^{-2}$ to $10^{17} \mathrm{Ga}^{+} \mathrm{cm}^{-2}$ across the rim), and the shape anisotropy factor is assumed to be constant in the entire sensing layer from exchange interactions. The relationship between $A_{C}$ and $A_{R}$ is calculated from equation 2 by 
comparing the results for the centre and the rim according to

$$
A_{C} / A_{R}=T_{R} R_{R} \int_{C} / T_{C} R_{C} \int_{R} .
$$

Finally, the width, $w$, of the rim can be calculated for the elliptical sensing layer which has a minor semi-axis, $r$, of $0.5 \mu \mathrm{m}$ and a two times larger major semi-axis, figure 5 , by inserting the expressions for the areas, $A_{C}=\pi(2 r-w)(r-w)$ and $A_{R}=2 \pi r^{2}-\pi(2 r-w)(r-w)$, into equation 3 giving

$$
w / r=3 / 2 \pm \sqrt{9 / 4-2 /\left(T_{R} R_{R} \int_{C} / T_{C} R_{C} \int_{R}+1\right)},
$$

with the constraint that $r>w$. Inserting the approximated data gives a rim width of $w=160 \mathrm{~nm}$, which sets a lower size limit to the fabrication process. Although, some approximations are necessary, this is probably the only way of estimating the size of the radiation damaged part of the junction, since no analysis method seems to have both the resolution and detection limit required for measuring it directly.



Figure 5. Geometry and interpolated hard axis TMR loops of the centre and rim.

The magnetization reversal of the sensing layer along the easy axis, figure 6 , was more straightforward, only showing more coercivity than an unirradiated junction from the reference batch. To investigate how the complexity of the sensing layer coercivity influenced the TMR, the magnetization reversal of the sensing layer was simulated in OOMMF, inset of figure 6. Both an unirradiated sensing layer and one with an irradiated rim were simulated. In the latter case, the sensing layer was given the same geometry as in figure 5 with $w=160 \mathrm{~nm}, T_{R}=0.4 T_{C}$ and $M_{S, R}=2 M_{S, C}$. It was seen that even though the softer centre of the sensing layer contributed to most of the TMR, the coercivity increased due to interactions between the rim and the centre, delaying the magnetization reversal of the latter part. 




Figure 6. Easy axis TMR loop, with inset showing the simulated TMR loops, for an irradiated and an unirradiated MTJ, respectively.

It should be said that the Ga implantation in the rim could be limited to a smaller width by, for instance, adding an extra step of pre-coating of the whole chip with an $\mathrm{Al}$ implantation barrier, which would absorb the Ga ion in the flanks of the Gaussian beam. However, this would increase the milling time for contour and bottom contact milling, figure 1 (c) and (e). Remembering that the process scheme presented here is intended for rapid prototyping, the applicability of this measure has to be weighed against the increase of the total process time.

The fabricated junctions had a TMR of $2.2 \%$ and a resistance-area product of $841 \Omega \mu \mathrm{m}^{2}$. The low TMR value was mostly due to the relatively high resistance of the bottom contact - typically around $11 \Omega$ as compared to $0.3 \Omega$ in the unirradiated junctions. The resistance of the bottom contact could be reduced by, e.g., making it smaller (reducing the distance between the four-point probes) or by reducing the dose rate (giving a smother milled surface). However, the former would reduce the flexibility of the scheme for the experiments in terms of the shapes and the number of junctions that can be patterned, and the latter would increase the process time. With a bottom contact resistance similar to that of the unirradiated junctions, the $T M R=\left(R_{\max }-R_{\min }\right) / 0.3 \Omega$, would have been $83 \%$, and the TMR of the centre, $T_{C}=1.48 T_{\text {mean }}$, would have been $123 \%$.

\section{Conclusion}

A rapid prototyping scheme for MTJs using FIB has been investigated, evaluated, and presented. MTJs of micrometer size were fabricated with high lateral resolution. The depth resolution was appropriately high thanks to the EPD etch stop. The MTJs showed some signs of magnetic degradation which was attributed to Ga implantation from the ion beam along the rim of the junctions, causing hysteresis in both the easy and hard axis TMR loops. The width of the irradiated rim was estimated from the hard axis TMR loop, using both theory and numerical interpolation, to $160 \mathrm{~nm}$, which sets a lower limit to the lateral size of junctions fabricated using this scheme. The bottom contact capping of the MTJs turned out to require an extra process step of Ar ion milling to remove the unwanted thin insulating $\mathrm{SiO}_{2}$ layer on top of the MTJs. In addition to the high resolution, there are two major advantages of particular importance for rapid prototyping: First, the versatility of the process makes it applicable to any MTJ stack - even to stacks comprised of materials prone to forming oxides since all process steps up to the deposition of the contacts can be performed in unbroken vacuum. Second, the simplicity and speed of the process makes it possible to go from design to a characterized MTJ in just a few hours making it an excellent tool for researchers.

\section{Acknowledgements}


The funding from the Swedish National Space Board, and the Swedish Governmental Agency for Innovation Systems, VINNOVA, is greatly acknowledged. The authors are also grateful to J. Langer and B. Ocker from Singulus AG for providing the TMR layer stacks, and to Klas Gunnarsson at the Dept. of Engineering Sciences and Vassilios Kapaklis at the Dept. of Physics at Uppsala University for help with the MFM and TMR measurements, respectively. IOP is greatly acknowledged for allowing the manuscript to be archived.

\section{References}

[1] Gallagher W J and Parkin S S P 2006 Development of the magnetic tunnel junction MRAM at IBM: From first junctions to a 16-Mb MRAM demonstrator chip IBM Journal of Research and Development 50 5-23

[2] Sining M, Nowak J, Dian S, Kolbo P, Lei W, Linville E, Saunders D, Murdock E, and Ryan P 2002 Spin tunneling heads above $20 \mathrm{~Gb} /$ in $^{2}$ IEEE Transactions on Magnetics 38 78-83

[3] Freitas P P, Ferreira R, Cardoso S, and Cardoso F 2007 Magnetoresistive sensors Journal of Physics: Condensed Matter 19165221

[4] Cyrille M C, Dill F, Jay L, Fontana R, Pinarbasi M, Baer A, Katine J, Driskill-Smith A, Jayasekara W, Mauri D, Ho M, Mackay K, and Ching T 2006 Nano processing strategies for MR sensor read width and stripe height formation IEEE Transactions on Magnetics 42 2434-7

[5] Gallagher W J, Parkin S S P, Lu Y, Bian X P, Marley A, Roche K P, Altman R A, Rishton S A, Jahnes C, Shaw T M, and Xiao G 1997 Microstructured magnetic tunnel junctions (invited) The 41st annual conference on magnetism and magnetic materials Atlanta Georgia (USA) 3741-3746

[6] Lee Y M, Ando Y, Miyazaki T, and Kubota H 2007 Reduction of switching fields of submicrometer sized magnetic tunnel junction with $\mathrm{NiFe}$-based synthetic ferrimagnetic free layer Journal of Applied Physics 101023905

[7] Fukushima A, Kubota H, Yuasa S, Takahachi T, Kadoriku S, and Miyake K 2007 Lift-off process for deep-submicron-size junctions using supercritical $\mathrm{CO}_{2}$ Journal of Magnetism and Magnetic Materials 310 687-689

[8] Rishton S A, Lu Y, Altman R A, Marley A C, Bian X P, Jahnes C, Viswanathan R, Xiao G, Gallagher W J, and Parkin S S P 1997 Magnetic tunnel junctions fabricated at tenth-micron dimensions by electron beam lithography Microelectronic Engineering 35 249-252

[9] Tsuboi S, Matsutera H, Ishi T, Ishiwata N, and Ohashi K $2001 \mathrm{Read} /$ write characteristics of focused-ion-beam-etched heads for perpendicular magnetic recording media Journal of Magnetism and Magnetic Materials 235 375-381

[10] Wei H X, Wang T X, Zeng Z M, Zhang X Q, Zhao J, and Han X F 2006 Controlled fabrication of nano-scale double barrier magnetic tunnel junctions using focused ion beam milling method Journal of Magnetism and Magnetic Materials 303 208-211

[11] Watanabe D, Kubota H, Ando Y, and Miyazaki T 2004 A simple fabrication process using focused ion beam for deep submicron magnetic tunnel junctions Japanese Journal of Applied Physics 43 7489-90

[12] Kaminsky W M, Jones G A C, Patel N K, Booij W E, Blamire M G, Gardiner S M, Xu Y B, and Bland J A C 2001 Patterning ferromagnetism in $\mathrm{Ni}_{80} \mathrm{Fe}_{20}$ films via $\mathrm{Ga}^{+}$ion irradiation Applied Physics Letters 78 1589-91

[13] Ozkaya D, Langford R M, Chan W L, and Petford-Long A K 2002 Effect of Ga implantation on the magnetic properties of permalloy thin films Journal of Applied Physics 91 9937-42

[14] Xiong G, Allwood D A, Cooke M D, and Cowburn R P 2001 Magnetic nanoelements for magnetoelectronics made by focused-ion-beam milling Applied Physics Letters 79 3461-3 
[15] Persson A, Nguyen H, Riddar F, and Thornell G 2009 Micro- and nanostructured magnetic field sensor for space applications 15th International Conference on Solid-State Sensors, Actuators and Microsystems. Transducers 2009 USA 1190-3.

[16] Telari K A, Rogers B R, Fang H, Shen L, Weller R A, and Braski D N 2002 Characterization of platinum films deposited by focused ion beam-assisted chemical vapor deposition Journal of Vacuum Science Technology B 20 590-5

[17] Donahue M J and Porter D G 1999 OOMMF User's Guide, Version 1.0 National Institute of Standards and Technology Gaithersburg, MD

[18] Chen C C, Lin J Y, Horng L, Yang J S, Isogami S, Tsunoda M, Takahashi M, and Wu J C 2009 Investigation on the magnetization reversal of nanostructured magnetic tunnel junction rings IEEE Transactions on Magnetics 45 3546-9 